\title{
A Possible Cure Therapy for Covid-19 Virus and Other Types of Viral Diseases. Using Autologous Stem Cell Trapping Mechanism
}

\author{
Amen Ugochukwu Ikeh* \\ Bsc Industrial Technical Education, University of Nigeria, Nsukka, Nigeria
}

\begin{abstract}
This research opinion paper advocates for a new system of treating viral infections and diseases, it lays more emphasis on the current virus called Covid -19 virus (Corona Virus), it is a new innovation different from the known methods of treating viral infections and diseases. Some of the known methods include use of vaccines, anti -viral medication, monoclonal, serology. Most recently, stem cells have proved to be very effective in solving complex medical diseases such as leukemia, cancer and a host of other medical diseases that pose difficult challenges to the medical field. This method could be used to cure covid-19 permanently and then simulated to solve and cure other viral infections such as HIV and AIDS, Ebola, Hanta Virus and a host of other diseases like Malaria, Chicken-Pox, Small Pox, Measles etc. It is worthy to note that viruses are always evolving by way of mutation and are developing intelligent and strategic ways to beat or avoid being detected by the immune system, this can be seen in HIV and AIDS virus which attacks CD4 cells and even goes as far as engaging and fighting to wear down the T-Cells. Also, Covid -19 tries to proofread the cells to counter anti-viral drugs such as Ribavirin. Although Remdesivir anti-viral medication which has proven to work on some covid-19 patients, cannot be proofread by the corona virus. More insight about this "Autologous stem cells trapping mechanism" is discussed under conceptual framework of this research paper and attention should also be given to the suggestions and recommendations as they hold potential methods to permanently stop the actions of viruses in invading the human cells.
\end{abstract}

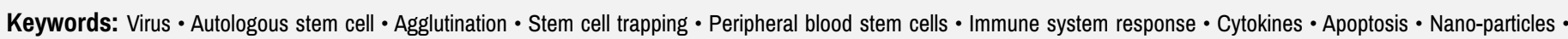
Monoclonal $\cdot$ Antibodies $\cdot$ Centrifuge $\cdot$ Serology $\cdot$ Vaccines

\section{Introduction}

\section{Background of the Study}

Fungi, bacteria and viruses immediately it is mentioned, the common perception to a layman is that all of them are harmful. In essence not every fungi, bacteria, virus are harmful and deadly. Some of them, especially viruses, are used in medical research positively. Some bacteria and viruses protect their host against other infections while others, especially viruses, are used in the process of evolution by helping to transfer genes among different species. In bio-medical research, scientists use virus to insert genes into cells. Unlike human cells or bacteria, virus do not contain the chemical machinery (enzymes) needed to carry out the chemical reactions for life. Instead viruses carry only one or two enzymes that decode their genetic instructions. Certainly a virus must have a host cell (bacteria, plant or animal) in which it can reside or co-habit and make more viruses. Outside of a host cell, it cannot function for this reason, virus threads the fine line that separates living things and non-living things [1].

Viruses are very small organisms with a generally simple and static structure. They have no metabolic system of their own. They depend on the machinery of their host cell for replication. Some viruses infect prokaryotic cells while others infect eukaryotic cells. Some viruses destroy cells, producing disease; others persist in infected cells either in a latent or persistent state; and other may cause cellular malignant transformation.

\section{Viral Structure}

Viruses are minimally composed of a nucleic acid genome (DNA or RNA) and a protein coat. Many viruses contain an external membrane called an envelope.

*Address for Correspondence: Amen Ugochukwu lkeh, Bsc. Industrial Technical Education, University of Nigeria, Nsukka, Nigeria, Tel: +2348188913232, E-mail: Ikehamen@gmail.com

Copyright: ( 2020 Ikeh AU. This is an open-access article distributed under the terms of the Creative Commons Attribution License, which permits unrestricted use, distribution, and reproduction in any medium, provided the original author and source are credited.

Received 14 July 2020; Accepted 29 July 2020; Published 05 August 2020
- The protein coat, or capsid, of an individual virion (fully assembled virus or virus particle) is composed of multiple copies of one or more types of proteins. These proteins assemble, forming structural units called Capsomeres.

- The nucleic acid plus the capsid shell of a virus particle is often called Nucleocapsid.

- The simplest viruses are those devoid of envelope with singlestranded DNA or RNA.

- Enveloped viruses contain an external membrane surrounding the nucleocapsid. The viral envelope is derived from host cell membranes (nuclear, Golgi apparatus, endoplasmic reticulum or plasma membrane). As such, it is composed by a lipid bilayer, with virus-encoded proteins inserted on it.

- Some viruses, such as Bacteriophages, have complex protein tails that are required for attachment and/or penetration of viral DNA into susceptible host cells.

Five Basic Structural Forms:

Based upon basic morphology, as indicated above, there are five different basic structural forms of viruses. These forms are listed below with examples:

- Naked Icosahedral - Adenoviruses and Picornaviruses.

- Naked helical - tobacco mosaic virus; no known human or animal viruses have this structure.

- Enveloped Icosahedral - Togaviruses and Flaviviruses.

- Enveloped helical - Rhabdoviruses and Paramyxoviruses.

- Complex - Bacteriophages and Poxviruses.

\section{How virus cause diseases}

Replication of the virus depends on the host cells chemical machinery. The steps may differ slightly depending on the type of host cell that the virus is attacking. Viruses lie around our environment all of the time just waiting for a host cell to come along. They can enter us through the eyes, nose, 
mouth or breaks in the skin. Once inside they find a host cell to infect. For example the cold and flu virus will attack the cells that line the respiratory or digestive tract [2]. system.

HIV which causes AIDS attacks T-cells (CD4 Cells) of the immune

- Ebola attacks the Macrophages and Dendritic Immune Cells; Small Pox attaches itself to Macrophages (Endothelial Cells) in the Liver, Spleen, Lymph Nodes and Bones.

- Measles attacks the macrophages and Dendritic Immune Cells.

- Covid-19 attacks ACE2 cell receptor (ACE2pathway) Angiotensin Converting Enzymes 2, are cell receptors that line Cells of Lungs, The Kidney, The Liver, The Aveoli Lining and Part of The Brain Cells.

When a virus infects a person (host), it invades the cells of its host in order to survive and replicate. Once inside, the cells of the immune system cannot 'see' the virus and therefore do not know that the host cell is infected. To overcome this, cells employ a system that allows them to show other cells what is inside them - they use molecules called class I Major Histocompatibility Complex Proteins (or MHC class I, for short) to display pieces of protein from inside the cell upon the cell surface. If the cell is infected with a virus, these pieces of peptide will include fragments of proteins made by the virus [3].

A special cell of the immune system called a T cell circulates looking for infections. One type of T cell is called a Cytotoxic T-cell because it kills cells that are infected with viruses with toxic mediators. Cytotoxic T cells have specialized proteins on their surface that help them to recognize virally-infected cells. These proteins are called T-Cell Receptors (TCRs). Each Cytotoxic T-cell has a TCR that can specifically recognize a particular antigenic peptide bound to an MHC molecule. If the T cell receptor detects a peptide from a virus, it warns its $T$ cell of an infection. The $T$ cell releases cytotoxic factors to kill the infected cell and, therefore, prevent survival of the invading virus [4].

Viruses are highly adaptable and have developed intelligent ways to avoid detection by T-cells, some viruses stop Macro-Histocompatibility Complex (MHC) molecules from getting to the surface to display viral peptides. If this happens the T-cells doesn't know there is a virus inside the infected cell. However, another immune cell specialize in killing cells that have a reduced number of MHC class1 molecules on their surfaces this cells are called Natural killer, NK cell. When the NK cell finds cells displaying fewer than normal MHC molecules it release toxic substances in a similar way to Cytotoxic T-cells which kill virally infected cell [5].

Cytotoxic cells are armed with preformed mediators. Cytotoxic factors are stored inside compartment called GRANULES in both cytotoxic T-cells and NK cells unit; contact with an infected cell triggers their release. One of these mediators is PERFORIN; a protein that can make pores in a cell membranes, these pores allows entry of other factors into a target cell to facilitate destruction of the infected cell. Enzymes called GRANZYMES are also stored in and released from the granules. Granzymes enter target cells through the holes made by perforin once inside the target cell, they initiate a process known as programmed cell death or apoptosis causing the target cell to die

Another released cyto-toxic factor is granulysin which directly attacks the outer membrane of the target cell destroying it by lysis. Cytotoxic cells also synthesize and release other proteins called cytokines after making contact with infected cells. Cytokines include interferon-alpha, and tumor necrosis factor-alpha, potentially priming a transfer signal from the T- cells to the infected or other neighboring cells, to enhance the killing mechanism. Once inside the target cell, they initiate a process known as programmed cell death or apoptosis, causing the target cell to die.

- Regardless of the type of host cell, viruses follow the same basic principle or steps to relocate:
- A virus particle attacks to a host cell.

- The particles release its genetic instruction into the host cell.

- The injected genetic materials recruit the host cells enzyme.

- The enzyme makes parts for more new virus particles.

- The new particles assemble the part into new viruses.

- The new particles break free from the host cell and spread into other healthy cells, repeating the same process.

All viruses have some type of protein on the outside coat or envelope that "feels" or "recognizes" the proper host cells. The protein attaches the virus to the membrane of the host cell. Some enveloped viruses can dissolve right through the cell membrane of the host because both the virus envelope and cell membrane are made of lipids. Those viruses that do not enter the cell must inject their contents (genetic instructions enzymes) into the host cell. Those viruses that dissolve into a cell simply release their contents once inside the host. In either case the result is the same [6].

\section{Abridged study explaining the (SARS-CoV-2) Coronavirus process of infecting the human body cells.}

The virus that causes Covid 19 is currently spreading around the world. Atleast six other types of coronavirus are known to infect humans, with some causing the common cold and two causing outbreaks: SARS and MERS.

\section{Covered with Spikes}

The coronavirus is named after the crown like spikes that protrude from its surface. The virus is enveloped in a bubble of oily lipid molecules, which falls apart on contact with soap.

\section{Entering a Vulnerable Cell}

The virus enters the body through the nose, mouth or eyes, then attaches to cells in the air way that produce a protein called ACE2. The virus is believed to have originated in bats, where it may have attached to as similar protein.

\section{Releasing Viral RNA}

The virus infects the cell by fusing its oily membrane with the membrane of the cell. Once inside, the coronavirus releases as nippet of genetic material called RNA.

\section{Hijacking the Cell}

The virus's genome is less than 30,000 genetic "letters" long. (Ours is over 3 billion.) The infected cell reads the RNA and begins making proteins that will keep the immune system at bay and help assemble new copies of the virus. Antibiotics kill bacteria and do not work against viruses. But researchers are testing antiviral drugs that might disrupt viral proteins and stop the infection.

\section{Making Viral Proteins}

As the infection progresses, the machinery of the cell begins to churn out new spikes and other proteins that will form more copies of the coronavirus.

\section{Assembling New Copies}

New copies of the virus are assembled and carried to the outer edges of the cell.

\section{Spreading the Infection}

Each infected cell can release millions of copies of the virus before the cell finally breaks down and dies. The viruses may infect nearby cells, or end up in droplets that escape the lungs

\section{Immune Response}

Most Covid 19 infections cause a fever as the immune system fights 
to clear the virus. In severe cases, the immune system can over react and start attacking lung cells. The lungs become obstructed with fluid and dying cells, making it difficult to breathe. A small percentage of infections can lead to acute respiratory distress syndrome, and possibly death.

\section{Leaving the Body}

Coughing and sneezing can expel virus laden droplets onto nearby people and surfaces, where the virus can remain infectious for several hours to several days. The C.D.C. recommends that people diagnosed with Covid 19 wear masks to reduce the release of viruses. Health care workers and others who care for infected people should wear masks, too.

\section{Roles of Cytokines (Definition, Types, Functions) - In inflammation}

Generally, cytokines are a variety of regulatory/signaling molecules (small proteins or glycoprotein) produced by various nucleated cells in the body. As products of nucleated cells (various cells of the immune system), they play a crucial role in the regulation of various aspects of immunity including the intensity and duration of immune responses by immune cells, etc. Based on studies, cytokines have also been shown to be pleiotropic and therefore have varying impacts on different types of cells. However, different types can also influence the activities of others and vice versa.

In addition to mediating and regulating immune responses, cytokines are also involved in the production and development of all the different types of blood cells (through a process known as Hematopoiesis), angiogenesis as well as Tumorigenesis among a few other important functions. Because of their role in body immunity, cytokines are also being used more as diagnostic and therapeutic agents [7].

\section{Types of Cytokines}

- Interferons

- Chemokines

- Interleukins (many interleukins are considered to be Lymphokines)

- TNF (Tumor Necrosis Factor)

\section{Interleukins}

Functions of Interleukins: Produced by such Leukocytes as Lymphocytes and Monocytes (and a number of other cells in the body e.g.) Interleukins are Glycoproteins involved in the activation and differentiation of immune cells. In addition, they also play an important role in the proliferation, migration, maturation, pro- and anti-inflammatory activities, as well as adhesion of these cells.

Together with interleukin receptors, interleukins belong to a superfamily (IL-Superfamily) that is made up of proteins. While it's easy to generalize the functions of these cytokines, this is largely dependent on the type of interleukins. Currently, over 43 members of this superfamily have been identified (IL-1 to IL-43) [8].

Like some of the other cytokines, interleukins are made up of proteins. Typically, this is in response to invading pathogens/antigens. While high volumes may be produced depending on the type of interleukins and the invading organism, a small quantity of the molecule is required to activate biological effects.

While a good number of interleukins elicit an action on the same cells that produced them, some can enter the bloodstream which allows them to be transported and elicit biological effects on distant cells in the body. For instance, while IL-2 elicits biological effects on T cells (which produced them), IL-1 can enter the bloodstream and reach the central nervous system

\section{Some of the other properties of interleukins include:}

- Synthesis is a self-limited process

\section{- Stimulate up-regulatory and down-regulatory mechanisms}

- Can influence the synthesis and functioning of other interleukins

\section{Interferons (IFNs)}

Interferon is a family consisting of widely expressed signaling proteins. Like the other cytokines, interferons are also released by cells of the host's immune system in response to such invading organisms as bacteria and viruses.

Virally infected cells produce and release small proteins called Interferons, which play a role in immune protection against viruses. Interferons prevent replication of viruses, by directly interfering with their ability to replicate within an infected cell. They also act as signaling molecules that allow infected cells to warn nearby cells of a viral presence - this signal makes neighboring cells increase the numbers of MHC class I molecules upon their surfaces, so that $T$ cells surveying the area can identify and eliminate the viral infection as described above [9].

They are also released to respond to tumorous cells in some organisms. Currently, three types of interferons have been identified. These include Type I IFNs, Type II IFN, and Type III IFNs.

Type I IFNs (Type I Interferons): Type I Interferons are divided into two major groups that include IFN- $\alpha$ and IFN- $\beta$ as well as a number of additional isotypes that include, among others, IFN- $\kappa$, IFN- $\omega$, and IFN- $\delta$. While only one type of IFN- $\beta$ exists, IFN- $\alpha$ is further divided into several subtypes including IFN- $\alpha 1$, IFN- $\alpha 2$, IFN- $\alpha 3$, IFN- $\alpha 4$, IFN- $\alpha 5$, and IFN- $\alpha 6$ among others.

In the body, the production of Type I IFNs is dependent on the presence of various microorganisms. For instance, following a viral infection, a signaling pathway that causes Phosphorylation, Dimerization as well as passage of the Interferon Response Factor 3 (IRF3) to the nucleus is activated [10].

Along with a number of other transcription factors, IRF3 activates that synthesis of IFN- $\beta$ gene that binds to the interferon receptors located on the surface of an infected cell which ultimately results in interferon response.

Through these responses, interferons help in the recruitment of effector molecules that protect the cells against infections (Bacterial and Viral Infections). For instance, by activating the production of natural killer cells and macrophages, interferons contribute to the destruction of both the viruses and infected cells.

Cells responsible for the production of IFN- $\alpha$ and IFN- $\beta$ are collectively known as Interferon-Producing Cells (IPCs) or Natural Interferon-Producing Cells [11].

Functions of Type I interferons: (IFN- $\alpha$ and IFN- $\beta$ ) are generally divided into three main categories that include:

Influence resistance to viral replication in cells - This is achieved through the destruction of viral mRNA required for viral replication as well as inhibiting the translation of viral proteins.

Promote ligand increase - Type-I Interferons promote an increase in ligands to the receptors of NK cells which in turn stimulates them to attack and lyse infected cells.

Activate the destruction of infected cells by NK cells and macrophages.

Given that Type 1 Interferons also play a role in immunosuppressive activities, they are also used for the purposes of treating autoimmune diseases.

Type II Interferon: Type II interferon is made up of a single cytokine known as IFN-y. This cytokine is largely produced by THI T cells, activated Natural Killer cells as well as CD8+ T cells. Unlike cytokines of Type I Interferons, the gene responsible for encoding the Type II cytokine is located in chromosome 12 in human beings [12].

Moreover, IFN-y has been shown to be different from the other 
interferons in that it does not produce a potent antiviral effect. Rather, it largely serves to activate Effector cells.

While it's produced by $T$ cells in adaptive immunity (following an increase in antigen), IFN-y is produced by natural killer cells in the innate immune system and acts as a mediator. In the adaptive immune system, increased production of IFN-y is promoted by IL-12 and IL-18.

On the other hand, IL-4 and IL-10 correspond to the negative regulators involved in its production. Apart from T cells, B cells and professional antigen-presenting cells have also been shown to play a role in the production of IFN-y.

Like cytokines of Type I Interferons, IFN-y also contributes to cell response to viral infections. For instance, by activating and causing the induction of MHC (Major Histocompatibility Complex), IFN-y has been shown to play a role in long-term control of viral infections in cells. In the process, it also coordinates the transition from innate to adaptive immunity [13].

Some of the other functions of IFN-y include: Macrophage activation - By promoting the activation of macrophages, IFN-y contributes to Phagocytic and Pinocytic activities of these cells and thus contributes to microbial destruction.

Inhibiting cell growth - In the body, IFN-y has also been shown to inhibit cell growth and thus promote apoptosis.

Type III Interferons: Unlike Type II interferon that only consists of a single cytokine, Type III is divided into three important cytokines that include IFN- $\lambda 1$, IFN- $\lambda 2$, and IFN- $\lambda 3$. Also known as IL-28 (a and b) and IL-29, Type III interferons are characterized by a structure that is more similar to proteins of the family IL- 10 .

On the other hand, the signaling pathway of these cytokines has been shown to resemble that of Type I interferons in that they are dependent on the actions of IRFs and NF-kB. While these cytokines also regulate a number of similar functions as Type I interferons, they primarily function in mucosal epithelial cells (as well as liver cells in human beings) [14], where they serve to protect them from viral infections.

\section{Chemokines}

Chemokines are a type of protein cytokine that play an important role in chemotaxis. As such, there may be signals that guide certain immune cells to the affected site.

Currently, about 50 chemokines have been identified.

While regulating leukocytes through the peripheral lymphoid tissues is one of the functions of chemokines, their primary role entails recruiting such cells as Neutrophils and T Cells to the site of inflammation.

Some of the other important functions of chemokines include:

- $\quad$ Promoting angiogenesis (CXC chemokines)

- Wound healing (CXC chemokines)

- Development of various non-lymphoid organs

- Priming certain T cells

For the most part chemokines are relatively small in size.

To achieve their functions, chemokines have to be released in large amounts so as to establish a concentration gradient necessary to influence cell migration.

\section{Tumor Necrosis Factor (TNF)}

Tumor Necrosis Factor (TNF) consists of a group of proteins involved in a number of physiological and pathological processes. Currently, about 40 members of the superfamily (TNF) have been identified with TNF- $\alpha$ and TNF- $\beta$ being the most notable examples.
TNF-alpha (TNF- $\alpha$ ): Also known as TNF or TNFSF2, TNF- $\alpha$ is a multifunctional cytokine involved in such processes as apoptosis and coagulation among others. In human beings, TNF- $\alpha$ gene is located on chromosome 6 . This allows the cytokine to be expressed and produced by such Immune Cells as Macrophages, Monocytes and T Cells in response to invading pathogens. Due to adipose tissue expression, TNF- $\alpha$ is sometimes referred to as an adipostat.

Functions of TNF- $\alpha$ : As mentioned, TNF- $\alpha$ is a multifunctional cytokine that ranges from immune roles to programmed cell death.

Immune function: Like the other cytokines, TNF- $\alpha$ plays an important role in immunity. In, TNF- $\alpha$ attracts certain immune cells to the affected site by stimulating the expression of adhesion molecules by vascular endothelial cells. This makes it possible for immune cells to adhere to blood vessel walls and successfully migrate to the infected site and destroy invading pathogens (Bacteria and Viruses) [15].

Induces the production of chemokines that are involved in inflammatory responses - These cytokines guide immune cells to the affected site.

Apoptosis: TNF- $\alpha$ promotes the programmed cell death of tumor cells by promoting the recruitment of proteins involved in death signaling.

Biological functions: When produced in large amounts, TNF- $\alpha$ has been shown to induce reduced blood pressure or shock during such events as severe infections. In some cases, however, a high concentration of this cytokine results in low blood sugar concentration as well as intravascular thrombosis.

TNF $\beta$ - Also known as Lymphotoxin: With regards to functions, TNF $\beta$ is a potent mediator involved in various immune and inflammatory responses that have similarities to those of TNF- $\alpha$.

Similar to TNF- $\alpha$, TNF- $\beta$ is also involved in the following processes:

- Apoptosis

- Coagulation

- Cell proliferation and differentiation

Receptors: Generally, receptors are membrane-bound molecules on which cytokines bind in order to elicit given cell functions. Therefore, cytokines can be said to influence cell functions/activities by binding to specific receptors.

Antibodies: Viruses can also be removed from the body by antibodies before they get the chance to infect a cell. Antibodies are proteins that specifically recognizes invading pathogens and bind (stick) to them. This binding serves many purposes in the eradication of the virus [16].

Firstly, the antibodies neutralize the virus meaning that it is no longer capable of inflecting the host cell.

Secondly, many antibodies can work together causing virus particles to stick together in a process called Agglutination. Agglutinated viruses make an easier target for immune cells than single viral particles.

A third mechanism used by antibodies to eradicate viruses, is the activation of phagocytes. A virus - bind antibody binds to receptors called $\mathrm{Fc}$, receptors on the surface of phagocytic cells and triggers a mechanism known as Phagocytosis, by which the cell engulfs and destroys the virus. Finally, Antibodies can also activates the complement system which Opsonizes and promotes Phagocytosis of viruses. Complement system can also damage the envelope (Phospholipid - Bilayer). That is present on some types of virus.

\section{Conceptual Framework}

\section{Autologous Stem Cell Trapping Mechanism}

This research article is advocating for the use of stem cell trapping 
PROCESS FLOW FOR A VIRUS TRAPPING MECHNISM

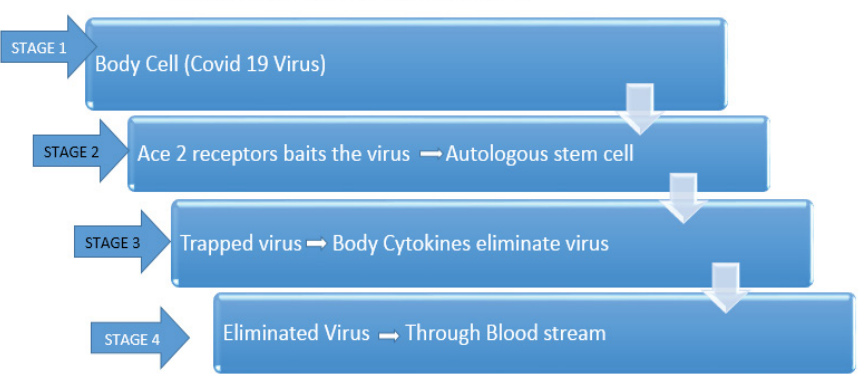

Figure 1. Diagram of Autologous Stem Cell Trapping Mechanism.

mechanism, it should be subjected to clinical test evaluation in order to ascertain its efficacy and effectiveness against Covid-19 Virus (Figure 1).

This stem cell trapping mechanism is referred to as a baiting technique. It is likened to the sugar ant baiting technique process, where sugar ants are being baited to become attracted and migrate towards the location where sugar is purposively placed. Similarly, this stem cell trapping technique is based on baiting the virus. For example, covid-19 virus loves ACE2 (ACE2 pathway), It loves going after angiotensin converting enzyme 2 cell receptor. Therefore, the stem cell should be coated with ace 2 receptor and it should be capable of emitting molecular aromatic flavor and signaling (smell, electrical or chemical attractant signaling) enabling the virus to move out from the infected cells in which they reside or cohabit and migrate towards the transplanted autologous stem cells. Purposively built inside, the stem cell should house body immune system molecules such as cytokines, chemokines, interleukins and interferons.

Once the virus enters into the autologous stem cell [17], through the ace 2 pathway, then primarily housed body cytokine molecules in the stem cell should be armed with sensory or perceptive inbuilt bio-sensor receptors that gets it triggered and released to effectively eliminate the virus. This technique; if really followed or expanded upon could be the effective cure therapy for eliminating viruses be it HIV, Ebola etc.

Its methodology hinge on tricking the virus to be displaced or dislodged out from the infected human immune cells and migrate towards the transplanted stem cells, which should preferably be autologous stem cells. Since the body defense mechanism does not fight against or resist autologous stem cells. More evidently, the human body immune system usually fights and offers resistance to foreign bodies that enters the body system. Basically, it does not see autologous stem cell as a foreign body. Moreover it essentially considers autologous stem cell, as majorly being a constituent member of the human body system. Consequently, causing the virus to migrate towards the autologous stem cells. As they find it irresistible not to get attracted or migrate towards it, then are eventually trapped and effectively eliminated with body cytokines housed in an inbuilt structure or compartment likened to granules in our body defense system. As earlier aforementioned in the research article, that agglutination process makes it is easier to eliminate viruses when many antibodies working together are involved, causing them to stick together a process known as Agglutination. This same similar principle is applied by gathering all the viruses together in a stem cell, then it can be eliminated with the effective and appropriate body cytokines. Some researchers always advocate for cell death or cell destruction known as apoptosis. This idea is good and brilliant, but reservation is expressed when you have too many viruses infesting an organism cells be it animal, plant or humans, and you try killing all the cells of the body, it will eventually lead to the death of that organism.

As earlier highlighted that attraction of viruses to a particular place makes it possible and easier for it to be eliminated as demonstrated by agglutination process, compared to when viruses are single and scattered. This opinion research paper strongly advocates for autologous stem cell to be used in the experimentation process. The autologous stem cells can be obtained from the, bone marrow, lymph nodes, spleen, thymus, and
Immune cells, such as T and B lymphocytes and macrophages are always negative for ACE2 cell receptors.

Preferably (Peripheral Blood Stem Cell) PBSC method or other effective methods of harnessing stem cells free from ethical issues should be incorporated. A critical survey of the recommendations and suggestions for further research study and highlighted below should be evaluated to see their possibility and implementation. More importantly it advocates for a semi-permeable cell- like membrane that has the ability to proof screen protein molecules and any other substances that enters the cell [18] (Figure 2).

\section{Mechanism of Suggested Proof Reading Semi-Permeable (Cell-Like) Membrane}

Recommendations for further research and study, is for a semipermeable cell-like membrane that can be grown over cell layers to act as a protective shield, that only allows air (Oxygen, Carbon Dioxide) and it is impenetrable to viruses, bacteria or fungi, for them to gain entry and access through it. Similarly it makes it difficult for them to wear down the semi-permeable cell-like membrane by injection of their viral molecular proteins into it, as these semi-permeable cell-like membranes should be able to proofread and screen protein molecules and selectively absorb only healthy protein molecules it recognizes, while others which it does not recognize will not be granted entry into the cell. If properly harnessed, this could stop some type of cancers caused by the actions of viruses. Potentially, it will act as a pre-regulator to cell activities, thus assisting in their healthy maintenance. In addition a molecule that could be injected that can lead to the stimulation of all body cells to grow this cell -like semi -permeable membrane. This semipermeable cell-like membrane growth should be encouraged after every virus in victims or host bodies have been successfully eliminated either through this stem cell technique or an expanded version of this technique [18].
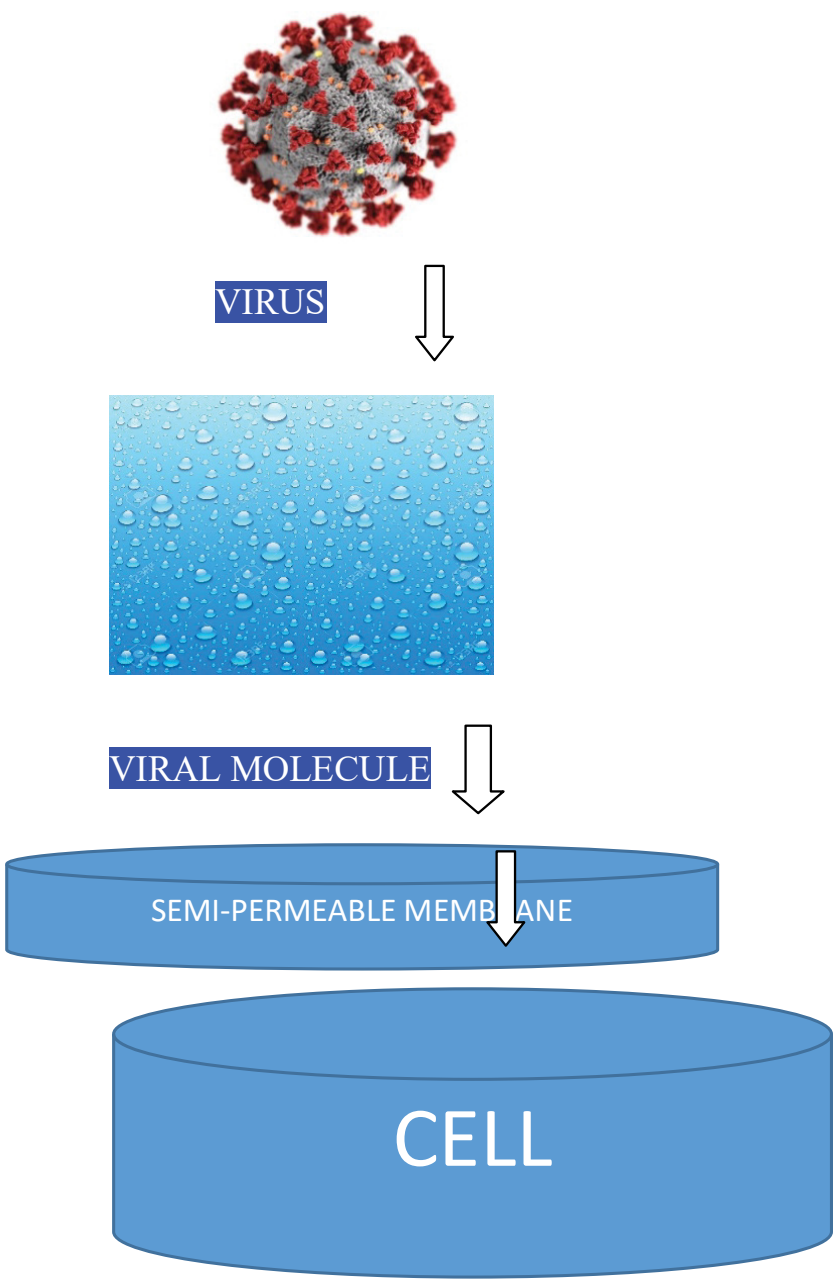

Figure 2. Diagram of Proof-Reading Semi - Permeable (Cell-Like) Membrane. 


\section{Theoretical framework}

Cytokine storm theory: Diseases such as covid-19 and influenza can be fatal due to an overreaction of the body's immune system called a cytokine storm. Cytokines are small proteins released by many different cells in the body, including those of the immune system where they coordinate the body's response against infection and trigger inflammation. The name 'cytokine' is derived from the Greek words for Cyto (cell) and Kinos (movement).

Sometimes the body's response to infection can go into overdrive. For example, when SARS -CoV-2-the virus behind the covid-19 pandemicenters the lungs, it triggers an immune response, attracting immune cells to the region to attack the virus, resulting in localized inflammation. But in some patients, excessive or uncontrolled levels of cytokines are released which then activate more immune cells, resulting in hyper inflammation. This can seriously harm or even kill the patient. Cytokine storms are a common complication not only of covid-19 and flu but of other respiratory diseases caused by corona viruses such as SARS and MERS. They are also associated with non-infectious diseases such as multiple sclerosis and pancreatitis. The phenomenon became more widely known after the 2005 outbreak of the avian H5N1 influenza virus, also known as "bird flu", when the high fatality rate was linked to an out-of-control cytokine response. Cytokine storms might explain why some people have a severe reaction to coronaviruses while others only experience mild symptoms. They could also be the reason why younger people are less affected, as their immune systems are less developed and so produce lower levels of inflammationdriving cytokines.

\section{Related empirical studies}

\section{Known methods of fighting viral diseases medically and their limitations}

Antibiotics, Anti-viral medications, Immunization and vaccines, Monoclonal, Serology:

- $\quad$ Antibiotics: Antibiotics are known to be effective on bacteria, but they are not effective on viruses.

- $\quad$ Anti-viral medications: Anti-viral medications mostly focus on symptom relief of viral infections, and do not fight against it. For example, cold medicine helps to alleviate the pain and congestion associated with the cold but it does not act directly on the cold virus. Anti-viral medications does not kill viruses they trap it, within 48 hours of early detection and sometimes due to the fact that some viruses change form or mutate, especially RNA viruses, it becomes less effective on the virus. Anti-viral drugs can equally damage the host cell where the virus resides. Another challenge with anti-viral drugs or medication is that a particular drug used to fight a particular virus may not be effective in fighting a new type of virus.

A very promising anti-viral drug strategy is using non-functional analogues or inauthentic copies of the basic building blocks of (RNA) genome. The presence of these analogues in the viral genome blocks the viral polymerase. Meaning the virus cannot make another copy of its (RNA), Acyclovir, Ribavirin and Azidothymidine (AZT) are examples of these anti-viral drugs. Unfortunately, the corona virus is a bit tricky because it "proofreads" the authenticity of its (RNA) genome. As such it identifies the analogues as being inauthentic and removes them. This stops certain anti-viral drugs like ribavirin from being effective. Fortunately, the corona virus proofreading powers do not block a similar anti-viral medication called remdesivir. So remdesivir potently halts corona virus replication and represents a promising drug option for covid-19 patients.

- Vaccines/Immunization: This works by pre-injecting the body so it knows how to produce the right antibodies as soon as the virus starts reproducing. Also, viruses reproduce fast and may change form and mutate so vaccines used earlier on may not be effective again and may require an upgraded form to be effective. Also vaccines are immune system boosters, that activate the white blood immune system to produce antibodies to fight off a viral infection, but sometimes even with vaccines a person's immune system may not be responsive enough quickly to produce the needed antibodies required to fight off a viral infection. Such factors that make it impossible for a person's immune system to respond quickly include age, heredity characteristics, etc. Vaccines are known to have caused side effects which are very harmful to the individual after administration. Some examples include brain damage (encephalitis) from small pox vaccine, sometimes vaccination does not kill the virus, as the virus may resist and even become more virulent, a good example is in the case of chicken pox transforming into shingles.

- Monoclonals: Antibodies are naturally produced by the immune system. However, scientists can produce antibodies in the lab that mimic the action of the immune system. These man-made (synthetic) antibodies act against proteins that attack normal tissues in people with auto-immune disorders. Man-made antibodies are produced by introducing human genes that produce antibodies into mice or another suitable mammal. The mice then are vaccinated with the antigen that scientists want to produce antibodies against. This causes the immune cells of the mice to produce the desired human antibody. The term monoclonal antibody means that the man-made antibody is synthesized from cloned immune cells, and the identical monoclonal antibody produced binds to one type of antigen. Polyclonal antibodies are synthesized from different immune cells and the antibodies produced bind to multiple antigens. Minor changes in antigen epitope structure affect the function of monoclonal.

- Serology: Blood serum is the clear liquid that remains after blood clots. Serum in a body is a component of plasma, as blood plasma is composed of a combination of both serum and coagulants. However, when separated from those coagulants through the use of a centrifuge, serum can be used to conduct a number of medical tests, and it can also be used to develop antiserum - used to help transfer resistance to disease from one body to another. A major disadvantage of serum is that they are scattered shot and they are not specific.

\section{Potential Medical Benefits of Autologous Stem Cell Trapping Mechanism}

- Trapping and eliminating the virus through autologous stem cells, assist in mutation challenges of viruses, caused by their resistance to vaccines, and antiviral medications.

- Introduction of semi -permeable membrane, which has the ability to proofread and screen protein molecules, allowing only those that are healthy and discarding toxic protein molecules. In addition making it impenetrable to breakdown by action of viruses or their molecular insertion into cells. This helps to curtail their disguise mechanism and render them latent.

- The semi - permeable membrane proof reading actions are not limited to viruses alone, as it aids to block other harmful and toxic microbes (bacteria, fungi.. etc) that will want to gain entry into the cell.

- Helps to regulate the challenge of under or over stimulation of body cytokine hormones, due to the controlled regulation of it through housed body cytokines in the transplanted autologous stem cells.

- The semi -permeable membrane also serves as pre-regulator to cells, thus assisting in the healthy functions of localized cells. 


\section{Summary}

Viruses are often used as vehicles for delivery in gene therapy because they're engineered not to damage the cell once they get there, but neglecting to consider how the virus will exit the cell which could have consequences. Some viruses use a molecule called heparin sulfate to help them attach to cells. These molecule, found in many different kinds of cells (including those from animal tissue), could prevent the virus from escaping. "It is not necessarily a good thing that the virus is not being released from the cell. That could have its own consequences on stimulating the immune system,", "As scientist are engineering viruses more and more to do gene transfer and gene therapy, a major factor is the need to consider their ability to exit the cell."Gene therapy is a relatively new technique that uses genes, rather than drugs or surgery, to treat a disorder. It can be done a few ways: by replacing a disease-causing gene with a healthy copy of the gene, knocking out a mutated gene that doesn't function correctly, or introducing an entirely new gene that would help the body fight disease.

Viruses used in gene delivery are engineered not to cause disease in a new host, but whether there will be unintended consequences of a virus never leaving the cell remains an unanswered question. "Heparin sulfate is potentially useful because rather than having the protein search for the cell's receptor in three-dimensional space, it brings the protein to the surface. Once visible, it makes the search two-dimensional, "But when we introduce the virus into a cell that makes heparin sulfate, it was not able to escape."Generating, healthy cells to replace diseased cells (regenerative medicine). Stem cells can be guided into becoming specific cells that can be used to regenerate and repair diseased or damaged tissues in people.

People who might benefit from stem cell therapies include those with spinal cord injuries, Type 1 Diabetes, Parkinson's Disease, Amyotrophic Lateral Sclerosis, Alzheimer's Disease, Heart Disease, Stroke, Burns, Cancer And Osteoarthritis. Stem cells may have the potential to be grown to become new tissue for use in transplant and regenerative medicine $[19,20]$.

\section{Conclusion}

The new system of permanently eliminating viruses is called the Autologous Stem Cell Trapping Mechanism. This method employs a cell baiting technique of attracting, trapping and then effectively eliminating the virus using any of the body cytokines, interferons or interleukins. This method could be used to cure covid-19 permanently and then simulated to solve and cure other viral infections such as HIV and AIDS, Ebola, Hanta virus and a host of other diseases like malaria, chicken-pox, small pox, measles etc. It is worthy to note that viruses are always evolving by way of mutation and are developing intelligent and strategic ways to beat or avoid being detected by the immune system. This can be seen in HIV and AIDS virus which attacks CD4 cells and even goes as far as engaging and fighting to wear down the T-CELLS, also covid-19 also tries to proof read the cells to counter anti-viral drugs such as Ribavirin. Although Remdesivir anti-viral medication which has proven to work on some covid-19 patient cannot be proof read by the corona virus. A good illustration about this autologous stem cell technique is likened to the process of trapping sugar ants by purposively placing sugar in specific locations where we want the migration of the sugar ants. Similarly, this stem cell method employs the same technique by coating autologous stem cells with cell receptors for example ACE2 cell receptor can be used for corona virus, since the virus is always attracted to ACE2 cell receptors. The stem cell being coated with Angiotensin Converting Enzyme (ACE2 cell) receptor and should provide an irresistible aroma or signal causing the virus to become attracted. It will cause the virus to be displaced out of the infected cells in which they reside and migrate towards the autologous stem cell transplanted. Recommendations are stated for further research and study to be carried out on a possible semi-permeable membrane cell-like receptor that can be grown over the cell acting as a shield that allows only air (oxygen, carbon dioxide) being impenetrable to viruses, bacteria or fungi, Similarly it makes it difficult for them to wear down these semi permeable membrane by injecting their viral molecular proteins into it, In addition these semipermeable membrane cell-like receptor should be able to proof read the protein molecules and selectively absorb only healthy protein molecules it recognizes while others it does not recognize are prevented from entering the cell.

From a theoretical point of view, stem cells are undifferentiated or partially differentiated cells meaning that cells in its raw form before it's differentiated into brain, liver, kidney cells etc. In line with our research, stem cells oppose viral infection due to the presence of specific cytokines improved qualities. These features are present in stem cells in the intrinsic niche before their separation process happens.

The pathogenesis is what has been explained "virus attaches to the ACE2 receptor".

ACE2 receptor is widely distributed on the surface of human cells, especially alveolar type 2 (AT2) and capillary epithelium. On the other hand, interestingly, bone marrow, lymph nodes, thymus, spleen, and immune cells, such as $T$ and $B$ Lymphocytes and Macrophages are always negative for ACE2.

These findings suggest that immunoglobulin therapy can help treat patients with the virus infection. There is much superiority in using stem cell therapy in comparison with other treatments. It has a high proliferation rate, low invasive procedure, and free of ethical issues. Importantly, you can easily get them from bone marrow, adipose tissue, dental pulp etc.

It is important to re-emphasize principally, that this research article is compiled to outline and provide a possible cure therapy towards diseases caused by viruses. Especially for the Covid -19 pandemic. The Covid-19 virus has resulted in the evident collapse of major economic activities of many countries around the world, in addition it has also resulted in the loss of jobs, and the unceasing increase in the number of death of humans. The author hereby implore the assistance of researchers, bio-medical engineers, bio-technologists, virologists together with other medical professionals and practitioners to work and expand upon the highlighted points contained in the conceptual framework, recommendations and suggestion for further findings of this research article.

\section{Recommendations}

Researches have been carried out by a company called IBM Company. Their fundamental research activities specialized in developing a macromolecule drug that uses electrostatic charge to attract viruses. Principally it has really been successful, although further research is still being undertaken by this company to see how it can effectively help humans. This principle can be transferred to the autologous stem cells that will assist to attract the virus from anywhere they are residing in the cell, in which they have infected. Moreover more innovative means or methods are welcomed, so long as the concept helps to lure the virus towards the autologous stem cell so that it could be trapped and eliminated effectively. The autologous stem cells should emit aromatic flavor or signals [21], so that the virus will find it irresistible and becomes attracted and migrate towards it. Evidently, when it enters the stem cell, it can be eliminated with any of the body cytokines, chemokines, interlukins, interferons, lympokines, monokynes.

An injection which could also be secreted, that has the potential to permeate through the body cells to drive out remnant viruses in order to displace them and support their migration to the autologous stem cell trap, where they are trapped, should be developed.

Recently a black African lady Hadiyah Nicole Green was able to use Nano particles to kill cancer cells in mice, using gold nano-particles. Similarly effective combination of nano-particles and autologous stem cell can be used as effective mechanism to eliminate viruses [22]. Since Nano particles avoid the use of Chemotherapy or radio radiation. As there are no side effects being recorded in humans. 
Nano particles have been used to boost stem cell growth and should be combined effectively for the enhanced production of stem cells. Innovative method of stem cell transplant that does not require chemotherapy or radiation should be embraced.

\section{Suggestions for Further Study and Research}

Furthermore, novel and innovative methods should be adopted to condense stem cells into suppositories so as to avoid exposing individuals to hazard of chemotherapy and radiation since it lowers the immune system response. Nano-particles could be used to guide the stem cells towards the site where they will be engrafted.

Categorically as ace 2 is coated on the surface of the autologous stem cell, in other to attract the Covid-19 virus and effectively eliminate it. Similarly, adequate cell receptors can be coated on other stem cells transplant for HIV, Ebola and Measles etc. As it is noted that all viruses or bacteria must have a particular cell receptor which they love and can penetrate it by using either their molecular insertion it or wearing down mechanism in other to access the cell $[23,24]$.

It was recently discovered that appendix which is one of the vestigial organs has the ability to host good bacteria. Similarly any of the vestigial organs such as the tailbone or wisdom tooth can be engrafted with stem cells that will have the ability to attract and capture harmful viruses. In other words, serving as a host organ for harmful viruses and equally equipped with cytokine molecules to eliminate it [25].

Recommendations for further research and study, is for a semipermeable cell-like membrane that can be grown over cell layers to act as a protective shield, that only allows air (oxygen, carbon dioxide) and it is impenetrable to viruses, bacteria or fungi, for them to gain entry and access through it. Irrespective of it being DNA or RNA viruses. Fundamentally making it difficult for them to wear down the semi-permeable cell-like membrane through the injection of their viral molecular proteins into it, since these semi-permeable cell-like membranes should be able to proofread and screen protein molecules and selectively absorb only healthy protein molecules it recognizes, while others which it does not recognize are not granted entry into the cell. If properly harnessed, this could stop some type of cancers caused by viruses. Essentially, it will be in form of a pre-regulator to cells activities thus assisting in their healthy maintenance. In addition a molecule that could be injected that can stir-up the stimulation of all body cells to grow this Cell -like semi -permeable membrane. This semipermeable cell-like membrane growth should be encouraged after every virus in victims or host bodies have been successfully eliminated either through this stem cell technique or an expanded version of this technique [26].

\section{Conflicts of Interest}

The author declare that there is no conflicts of interest regarding the publication of this article

\section{Authors' Contributions}

The under listed names contributed equally to this work

Dr. Abdulhakeem Sobodun, Sonaya Oluwadamilola Emmanuella, Dr. Ben Olusola , Dr. Sunny Oshodi, Dr. Toba Babarinsa, Ikeh Majesters Nnamdi, Eno Thompson Nse, Oluwadamilare Lukman Tijani, Engr. Matthew Amen Ikeh, Alaki Clifford, Opeyemi Olanite Ismail, Dr. Oluigbo Caleb, Nnaemeka Miracle, Udoka Nwabunike, Izuchukwu Modestus Ifeanyi, Mordi Chukwunonso, Adeleke Joshua Owolabi , Esther Tosin, Michael Nkordeh, Odu Paul, Ogundipe Benjamin, Gift Wuche, Chijioke Ifeoma Chiwendu, Bright Nwogu, Joan .C. Ananaba, Dr. Joe Ersinghaus, Kazeem Adebola Ibrahim, Folu john, Mrs.John Abosede, and the peer editorial review and management team of https://www.hilarispublisher.com/ bioanalysis-biomedicine.html for their resourceful advice and support.

\section{Acknowledgement}

Firstly, I want to acknowledge and appreciate God Almighty who divinely inspired me with this idea, and also giving me the strength to accomplish this research work, positively contributing my own little quota to the advancement, upliftment and betterment of humanity all to the honor and glory of God. Secondly, My sincere appreciation and acknowledgement goes to the underlisted names, As they all collectively supported and worked to see to the manifestation of this research opinion paper.

Dr. Ben Olusola, Gift Wuche, Esther Tosin, Chijioke Ifeoma Chiwendu, Oluwadamilare Lukman Tijani, Ikeh Majesters Nnamdi, Alaki Clifford Msuega, Mordi Chukwunonso, Udoka Nwabunike, Ogundipe Benjamin, Michael Nkordeh, Engr. Matthew Amen Ikeh, Dr. Abdulhakeem Sobodun, Sonaya Oluwadamilola Emmanuella, Kazeem Adebola Ibrahim, Adeleke Joshua Owolabi, Odu Paul, Olanite Opeyemi Ismail, Izuchukwu Modestus Ifeanyi, Bright Nwogu, Awoneye Temitope, Eno Thompson Nse, Dr. Toba Babarinsa, Dr. Oluigbo Caleb, Nnaemeka Miracle, Dr. Joe Ersinghaus, Dr. Sunny Oshodi, Joan .C. Ananaba, Folu john, Mrs. John Abosede, High Chief. Raphael Obiduba, Prof. Greg Simire, Mrs. Margaret ovonlen, Chief. Vitals Ibe, Mr. Nnaji Chimezie.

Special thanks and appreciation to the peer editorial review and management team of https://www.hilarispublisher.com/bioanalysisbiomedicine.html for their resourceful advice and support.

\section{References}

1. Michel, Roland. "Black Woman becomes the first doctor to cure cancer in mice using Nanoparticles". Black Enterprise articles 2008.

2. Buckner, $\mathrm{CD}$, and $\mathrm{CH}$ Weaver. "Autologous stem cell transplant". Cancer Connect 2020.

3. Cohen, John. "The race is on for antibodies that stops the new corona virus". Science Mag 2020.

4. "Importance of Stem Cells" University of Nebraska Medical Center 2020.

5. Edwards, Micheal. "How viruses work and how to prevent and eliminate them naturally". Urology of Virginia Articles 2014.

6. Liang, Kenny, and Fred Hutchnson. "Immune Responses to viruses". British society for Immunology Articles.

7. Freudenrich, Craig, and Patrick Kiger J "How viruses work". 2020

8. Thesis, Honors. "Ebola tissue tropism and pathogenesis". 1999.

9. Murell, Daniel. "How does HIV affect the body". Health Clinic Article 2020.

10. Taraban, Vardim, and Robie Baldock. "Corona Virus Cytokine storm: This over active Immune response could be behind some fatal cases of COVID-19." The conversation.com Articles 2020.

11. Wadman, Maredith, Jennifer Conzin, Frankel Joceyln Kaiser and Catherine Matacic, et al. "How does corona virus kill? Clinicians traced a ferocious rampage through the body from grain to toes." 2020

12. Flaherty, Julie. "How the body battles Covid-19." Tufts Now 2020.

13. Brumfiel, Geoff. "Why some Covid-19 patients crash: The body's immune system might be to blame" 2020 .

14. Estes, Clay. "What is cytokine storm and why is it so deadly for corona virus patients?". forbes articles 2020

15. George, Alison. "Cytokine Storm: and overreaction by the bodys immune system" 2020

16. Klimpel, Gary R. "CHAPTER 50 Immune defenses". The University of Texas Medical branch, Galveston, 1996.

17. Touchstone, Liz Ahlberg. "Nano stimulators boost stem cells for muscle repairs." 2020. 
18. Ugochukwu, Ikeh Amen. "A possible cure therapy for covid -19 virus and other types of viral diseases. Using autologous stem cell trapping mechanism." 2020.

19. Sonaya, OE. "A possible cure therapy for covid -19 virus and other types of viral diseases. Using autologous stem cell trapping mechanism". 2020.

20. Sobodun, AB. "A possible cure therapy for covid -19 virus and other types of viral diseases. Using autologous stem cell trapping mechanism." 2020.

21. Yoo, Min Hyuk, and Dolph Hatfield L. "The cancers stem cell theory: is it correct?". 2009.

22. Bardelli, Silvana, and Macro Moccetti. "Remodelling the human adult stem cell niche for regenerative medicine applications." 2014.

23. Liao, Yanling, Hongwen Zhu, Larissa Ivanava and Mitchell Cairo S, et al. "Innovations in human stem cell research: A Holy grail for regenerative medicine." 2019.
24. All day, Erin, Peter Hoey and Emma Neil O, et al. "What are stem cells and how do they work". San Francisco Chronicle 2018.

25. Cafasso, Jacquelyn. "Stem cell research". University of Illonois, Chicago, 2017.

26. Staff, Mayo Clinic. "Stem cells: what they are and what they do".

How to cite this article: Amen Ikeh Ugochukwu. "A Possible Cure Therapy for Covid-19 Virus and Other Types of Viral Diseases. Using Autologous Stem Cell Trapping Mechanism". J Bioanal Biomed 11 (2020) doi: 10.37421/ jbabms.2020.12.228 